\title{
Functional Cloning and Expression of eme $A$, and Characterization of EmeA, a Multidrug Efflux Pump from Enterococcus faecalis
}

\author{
Eun-Woo Lee, Jing Chen, Md. Nazmul Huda, Teruo Kuroda, Tohru Mizushima, and \\ Tomofusa TsuchiYA* \\ Department of Microbiology, Faculty of Pharmaceutical Sciences, Okayama University; Tsushima, Okayama 700-8530, \\ Japan. Received October 9, 2002; accepted November 30, 2002
}

\begin{abstract}
A fragment of chromosomal DNA from Enterococcus faecalis ATCC 29212 was cloned using Escherichia coli KAM32 host cells lacking major multidrug efflux pumps. $E$. coli KAM32 cells were sensitive to many antimicrobial agents, and the transformed cells harboring a recombinant plasmid became resistant to several structurally unrelated antimicrobial agents such as tetraphenylphosphonium chloride, 4',6-diamidino-2-phenylindole (DAPI), Hoechst 33342, acriflavine, benzalkonium chloride, norfloxacin and ethidium bromide. This suggests that the cloned DNA fragment carries a gene(s) encoding a multidrug efflux pump. Determination of the nucleotide sequence of the cloned DNA revealed a gene designated as eme $A$. The transformed $E$. coli cells showed efflux activity of several antimicrobial agents such as DAPI, Hoechst 33342 and acriflavine. Efflux of DAPI via EmeA was strongly inhibited by reserpine.
\end{abstract}

Key words EmeA multidrug efflux pump; drug efflux; drug resistance; Enterococcus faecalis

Over the past several decades, the emergence of drug resistance in microorganisms, and its association with serious infectious diseases, has increased at an alarming rate. ${ }^{1)}$ Many bacteria that once appeared to be under control or were believed to be potentially controllable are now causing infections that are increasingly difficult to treat. ${ }^{2)}$ There is a large reservoir of resistance genes in bacterial genomes, and in extra-chromosomal pieces of DNA, that are responsible for different mechanisms of drug resistance such as efflux pumps, enzymes that inactivate antibiotics and which alter antibiotic targets. ${ }^{3)}$ Among them, multidrug efflux pumps are emerging as a major problem in multidrug resistance in bacterial cells. ${ }^{4,5)}$

The extensive knowledge of the molecular mechanisms underlying microbial antibiotic resistance is required in order to successfully fight the increasing numbers of drug resistant and multidrug resistant (MDR) bacteria. ${ }^{3)}$ Thus, it is important to investigate the multidrug efflux pumps of bacteria at both the genetic and biochemical levels. Gene cloning, expression, and biochemical analysis are useful approaches to the understanding of the mechanism of multidrug efflux pumps. ${ }^{6}$ )

Enterococci, gram-positive and facultative anaerobic bacteria, are normally found in the human intestine. ${ }^{7}$ Over the past two decades, enterococci have been identified with increasing frequency as causative agents of nosocomial infections, paralleling the correlation of antimicrobial resistance to most commonly used agents. As a result, enterococci have emerged as one of the leading clinical challenges for physicians when identified as the cause of serious or life-threatening infections. ${ }^{8)}$ Infections caused by bacteria of the genus Enterococcus include urinary tract infections, nosocomial bacteremia, intra-abdominal infections and endocarditis. ${ }^{79}$ )

Most enterococci have intrinsic resistance to various antimicrobial agents, including $\beta$-lactams, fluoroquinolones, aminoglycosides and glycopeptides. ${ }^{10,11)}$ The rapid emergence of antimicrobial resistance among the enterococci undoubtedly also contributes to their emergence as prominent nosocomial pathogens, making them among the most diffi- cult to treat. It was hypothesized that resistance to multiple antimicrobial drugs of this strain probably explains to a large extent its prominence in nosocomial infections. ${ }^{12)}$

Unlike gram-negative enteric bacteria, an outer membrane does not protect the enterococcal cell. Lynch et al. hypothesized that, in the absence of an extracytoplasmic permeability barrier, the most likely mechanism for intrinsic drug resistance in enterococci may be active efflux. ${ }^{13)}$ Recently, Jonas et al. ${ }^{14)}$ reported PCR cloning of emeA gene encoding a multidrug efflux pump and some properties of the pump, from Enterococcus faecalis which causes most enterococcal infections in humans.

In this study, we report functional gene cloning and characterization of the EmeA as multidrug efflux pump, from $E$. faecalis. We found better substrates for the pump and measured their extrusion from cells.

\section{MATERIALS AND METHODS}

Bacteria and Growth E. faecalis ATCC 29212, Escherichia coli $\left.\operatorname{KAM} 32(\Delta a c r B, \Delta y d h E)^{6}\right)$ were used in this study. E. faecalis cells were grown in Brain Heart Infusion (BHI) medium (Difco Co.) and E. coli cells were grown in L medium ${ }^{15)}$ at $37^{\circ} \mathrm{C}$. Measurement of the optical density at $650 \mathrm{~nm}$ was used to monitor cell growth.

Cloning and Sequencing Chromosomal DNA was prepared from E. faecalis cells by the method of Berns and Thomas. ${ }^{16)}$ The DNA was partially digested with Sau3AI and the fragments from 4 to $10 \mathrm{kbp}$ were separated by sucrose density gradient centrifugation. Plasmid pBR322 DNA was digested with BamHI, dephosphorylated with bacterial alkaline phosphatase (BAP) and then ligated to the chromosomal DNA fragments by using a ligation kit, Ver. 2 (TaKaRa Co.). Competent cells of $E$. coli KAM32 were transformed with the recombinant plasmids and were spread onto agar plates containing L broth, $60 \mu \mathrm{g} / \mathrm{ml}$ ampicillin, $10 \mu \mathrm{g} / \mathrm{ml}$ tetraphenylphosphonium chloride (TPPCl) and $1.5 \%$ agar. The plates were incubated at $37^{\circ} \mathrm{C}$ for $24 \mathrm{~h}$. Candidate colonies were replica plated, and plasmids were isolated from each of 
the candidates. Plasmid DNAs were re-transformed into the KAM32 cells and spread onto the same type of plate. Plasmids were isolated from each of the retransformants and used for restriction mapping and sequencing. The nucleotide sequence of the gene was determined by the dideoxy chain termination method ${ }^{17)}$ with an automated DNA sequencer (ALF Express, Pharmacia Biotech.). The nucleotide sequence data has been deposited in the DDBJ/EMBL/GenBank nucleotide sequence databases with the accession number AB091338.

Drug Susceptibility Test The minimal inhibitory concentrations (MICs) of various drugs were determined in Muller-Hinton broth (Difco) by the standard 2-fold dilution method. ${ }^{18)}$ Cells were incubated in the test medium at $37^{\circ} \mathrm{C}$ for $24 \mathrm{~h}$ and the growth was examined by visual inspection.

Fluorometric Assay of EmeA-Mediated Efflux of Drugs Fluorometric assays of EmeA-mediated efflux of 4',6-diamidino-2-phenylindole (DAPI), Hoechst 33342 and acriflavine were carried out essentially as described previously for ethidium. ${ }^{19)}$ DAPI binds to double-stranded DNA, resulting in a substantial increase in fluorescence. ${ }^{20)}$ Hoechst 33342, when bound to membrane lipids and/or DNA, are highly fluorescent ${ }^{21)}$; acriflavine binds to DNA resulting in fluorescence quenching. $\left.{ }^{6}\right)$ E. coli KAM32 cells harboring recombinant plasmids were grown in $\mathrm{L}$ broth at $37^{\circ} \mathrm{C}$ to the exponential phase of growth. The cells were harvested and washed with modified Tanaka buffer $(\mathrm{pH} \mathrm{7.0)})^{22)}$ containing $2 \mathrm{~mm} \mathrm{MgSO}_{4}$. Cells were loaded with $4.2 \mu \mathrm{M}$ acriflavine by incubating for $1-2 \mathrm{~h}$ at $37^{\circ} \mathrm{C}$ in the presence of $40 \mu \mathrm{M}$ carbonyl cyanide $m$-chlorophenylhydrazone (CCCP). For loading of DAPI $(5 \mu \mathrm{M})$ or Hoechst $33342(1 \mu \mathrm{M})$, dinitrophenol (DNP, $5 \mathrm{~mm}$ ) instead of CCCP was used and incubated for $10 \mathrm{~h}$ at $37^{\circ} \mathrm{C}$. Cells were washed three times, and resuspended with $0.1 \mathrm{M}$ MOPS-tetramethylammonium hydroxide (TMAH) ( $\mathrm{pH} 7.0$ ) containing $2 \mathrm{mM} \mathrm{MgSO}_{4}, 20 \mathrm{~mm} \mathrm{KCl}$ and each substrate of the same concentration as indicated above. Energy-dependent efflux was assayed following the addition of glucose to a final concentration of $20 \mathrm{~mm}$. Fluorometric measurements were performed at $37^{\circ} \mathrm{C}$ using a Hitachi 2000 fluorescence spectrophotometer. The excitation and emission wavelengths used for each of the fluorescent compounds are as follows: DAPI, $332 \mathrm{~nm}$ and $463 \mathrm{~nm}$; Hoechst 33342 , $355 \mathrm{~nm}$ and $457 \mathrm{~nm}$; and acriflavine, $468 \mathrm{~nm}$ and $499 \mathrm{~nm}$, respectively.

\section{RESULTS}

Functional Cloning and Sequencing of the emeA DrugResistance Gene In order to clone an E. faecalis gene responsible for multidrug resistance, partially digested chromosomal DNA fragments from E. faecalis were ligated into the cloning vector pBR322, and transformed into $E$. coli KAM32 $(\Delta a c r B, \Delta y d h E)$. We obtained four recombinant plasmids using TPPCl as an antimicrobial agent for selection. Restriction analysis revealed that these four plasmids contained the same DNA region. One of them, pTEF7, was used for further analysis (Fig. 1). The insert of pTEF7 was about $2.9 \mathrm{kbp}$ long. Sequencing of the DNA insert in plasmid pTEF7 revealed that it contained two complete open reading frames (ORF1 and ORF2) and one of them, ORF2, was found to be almost same as eme $A^{14)}$ (locus EF1078), ${ }^{23)}$ which encodes a multidrug resistance protein.

We constructed several hybrid plasmids carrying various portions of the insert DNA region from pTEF7. The resulting hybrid plasmids were introduced into E. coli KAM32 cells, and the transformants were tested for sensitivity or resistance to TPPCl (Fig. 1). A plasmid pTEF71 contained the whole region of emeA and a part of ORF1. E. coli KAM32 cells harboring plasmid pTEF71 showed resistance to TPPCl. A plasmid pTEF72, which carried only emeA located downstream from the tet promoter of pBR322, conferred drug resistance. Plasmid pTEF73, carrying most of the eme $A$ gene, and plasmid pTEF78, carrying ORF1, did not confer drug resistance. Thus, it is clear that emeA alone is enough for the drug resistance if it is appropriately expressed.

We determined the nucleotide sequence of the whole eme $A$ region because we cloned the gene from $E$. faecalis ATCC 29212, not from E. faecalis V583. We found that there were some differences (37 different nucleotides at various positions) in the sequence between the eme $A$ gene of $E$. faecalis ATCC 29212 and the EF1078 locus of E. faecalis V583 ( $97 \%$ identity in the nucleotide sequence). The eme $A$ gene was preceded by a possible promoter-like sequence and a ribosome-binding sequence (Shine-Dalgarno sequence). The gene was followed by a putative transcription terminator-like (inverted repeat) sequence. The emeA gene, $1179 \mathrm{bp}$ in length, specifies a putative 393-amino acid protein with a calculated molecular mass of $42.1 \mathrm{kDa}$. The identity in the amino acid sequence between EmeA from strain ATCC29212 and V583 was $97.7 \%$. Only nine amino acid residues (at the position 141-149) were different. Hydropathy analysis ${ }^{24)}$ of EmeA predicted a highly hydrophobic protein with 12 membrane-spanning regions and hydrophilic sequences at both the $-\mathrm{NH}_{2}$ and $-\mathrm{COOH}$ termini of the protein (data not shown).

The deduced amino acid sequence of the EmeA showed $80,47,46$ and $35 \%$ identities and 89, 64, 66, and 61\% similarities with putative Blt of Lactococcus lactis, ${ }^{25)} \mathrm{Bmr}$ of Bacillus subtilis, ${ }^{26)}$ Blt of B. subtilis, ${ }^{27)}$ and NorA of Staphylococcus aureus, ${ }^{28)}$ respectively, as suggested by Jonas et al. ${ }^{14)}$

Drug Susceptibility Studies in $\boldsymbol{E}$. coli To define the contribution of EmeA towards mediating drug resistance, the

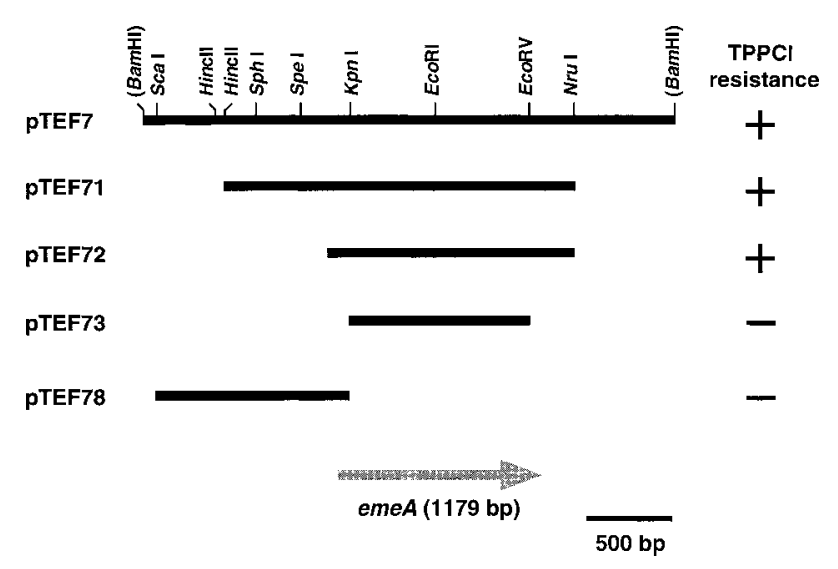

Fig. 1. Restriction Map of Plasmid pTEF7 and Its Derivatives

Horizontal bars indicate DNA regions derived from the chromosomal DNA of $E$. faecalis. Plus signs $(+)$ on the right hand side indicate that E. coli KAM32 cells harboring each plasmid grew on an L plate containing $10 \mu \mathrm{g} / \mathrm{ml} \mathrm{TPPCl}$ and $60 \mu \mathrm{g} / \mathrm{ml}$ ampicillin. Minus signs $(-)$ indicate that cells did not grow on the same plates. The arrow indicates the position and direction of the emeA gene. Restriction sites are shown at the top. 
protein was expressed in E. coli KAM32, which is hypersensitive to many drugs due to deficiencies of the major multidrug efflux pumps AcrAB and YdhE. ${ }^{6}$ ) The drug susceptibility in cells of KAM32/pTEF71 (carrying emeA) and KAM32/pBR322 (control) are shown in Table 1. Plasmid pTEF71 in KAM32 cells conferred resistance to several structurally unrelated drugs. The MICs of TPPCl, DAPI, Hoechst 33342, acriflavine, benzalkonium chloride, norfloxacin, ciprofloxacin, and ethidium bromide in E. coli KAM32/pTEF71 were 16-, 8-, 8-, 4-, 8-, 8-, 4-, and 2-fold higher, respectively, than the control. Thus, we conclude that the EmeA is a protein conferring multidrug resistance, perhaps a multidrug efflux pump. No significant change in the MIC was observed with the other drugs tested in this study (Table 1).

EmeA-Mediated Efflux of Various Fluorescent Antimicrobial Agents It has been reported that EmeA mediates efflux of ethidium from cells. ${ }^{14)}$ We further tested whether EmeA mediates efflux of structurally dissimilar compounds DAPI, Hoechst 33342 and acriflavine (Fig. 2). In the cases involving DAPI and Hoechst 33342, fluorescence of dilute solution increases when each are bound to DNA, ${ }^{20,21)}$ indicating that the fluorescent dye is accumulated in the cells. On the other hand, the fluorescence of acriflavine decreases when it binds to DNA. ${ }^{6}$ In Fig. 2, energy-dependent effluxes of DAPI and Hoechst 33342 are shown as rapid decrease in

Table 1. MICs of Various Drugs in E. coli KAM32 Harboring Either Control Plasmid or Plasmid Carrying emeA Gene

\begin{tabular}{lcc}
\hline \multirow{2}{*}{ Drug } & \multicolumn{2}{c}{ MICs $(\mu \mathrm{g} / \mathrm{ml})$} \\
\cline { 2 - 3 } & KAM32/pBR322 & KAM32/pTEF71 \\
\hline TPPCI & 8 & 128 \\
DAPI & $<0.06$ & 0.5 \\
Hoechst 33342 & 0.5 & 4 \\
Acriflavine & 1 & 4 \\
Benzalkonium & 0.015 & 0.12 \\
Ethidium bromide & 2 & 4 \\
Norfloxacin & 0.03 & 0.25 \\
Ciprofloxacin & 0.004 & 0.015 \\
Chloramphenicol & 1 & 1 \\
Erythromycin & 4 & 4 \\
Tetracycline & $0.5^{a)}$ & 0.5 \\
Rhodamine 6G & 8 & 8 \\
\hline
\end{tabular}

a) Value with KAM32 not harboring pBR322. their fluorescence intensities, whereas efflux of acriflavine is observed as a rapid increase in the fluorescence. These results showed that structurally different chemicals (at least three substrates listed here) were extruded via EmeA. Thus, EmeA is indeed a multiple drug efflux pump.

EmeA-mediated efflux of all three of the substrates tested was abolished by the addition of CCCP (data not shown), a proton conductor, suggesting that a proton-motive force drives the EmeA-mediated extrusion of several structurally dissimilar antimicrobial agents.

Subsequently, we tested the effect of monovalent cations, such as $\mathrm{Na}^{+}, \mathrm{Li}^{+}$or $\mathrm{K}^{+}$, in the efflux process because several multidrug efflux pumps are coupled with $\left.\mathrm{Na}^{+} .6,19,29\right)$ The addition of either $\mathrm{KCl}, \mathrm{NaCl}$ or $\mathrm{LiCl}$ showed no significant difference in the efflux activity compared to the control (data not shown). Thus, we conclude that the drug efflux via the EmeA pump is an $\mathrm{H}^{+}$-coupled one.

Inhibition of EmeA-Mediated Drug Efflux by Reserpine Reserpine is known as an inhibitor of a wide range of multidrug efflux pumps. These systems include P-glycoprotein $^{30)}$ (a member of the $\mathrm{ABC}$ super-family), Bmr from $B$. subtilis, ${ }^{31)}$ LmrP from L. lactis, ${ }^{32)}$ and NorA from S. aureus ${ }^{33)}$ (members of major facilitator superfamily (MFS)), and EmrE from $E$. coli $^{34)}$ (a member of small multidrug resistance super-family). Jonas et al. $^{14)}$ reported that reserpine (at 20 $\mu \mathrm{g} / \mathrm{ml}$; roughly $33 \mu \mathrm{M}$ ) only slightly inhibited efflux of ethidium via EmeA. We investigated the effect of reserpine on the EmeA-mediated efflux of DAPI. We observed a very strong inhibition of EmeA activity due to reserpine (Fig. 3). The concentration of reserpine giving $50 \%$ inhibition $\left(\mathrm{IC}_{50}\right)$ of the initial rate of EmeA-mediated DAPI efflux was calculated to be $15 \mu \mathrm{M}$. Similar strong inhibition by reserpine was observed with ethidium as an efflux substrate (data not shown). The reason for the difference with the observation by Jonas is not clear at the present time.

\section{DISCUSSION}

Recently, the nucleotide sequencing of the entire chromosome and plasmids of E. faecalis V583 has been completed and are available on the web. ${ }^{23)}$ Based on the sequence information, Jonas et al. ${ }^{14)}$ reported on the PCR cloning of a gene emeA encoding a multidrug efflux pump from E. faecalis strains OG1RF and V583, and reported some of the properties of the EmeA pump. They reported that EmeA was re-
A

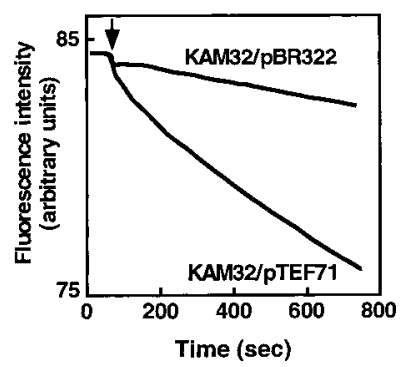

B

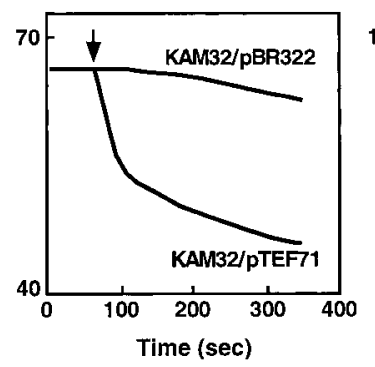

C

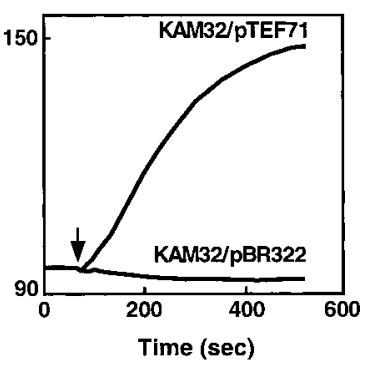

Fig. 2. Fluorometric Assays of Drug Efflux

Efflux of DAPI (A), Hoechst 33342 (B), and acriflavine (C) from cells of KAM32/pBR322 or cells of KAM32/pTEF71 (carrying emeA) were measured. Energy-starved cells were loaded either with DAPI, Hoechst 33342 or acriflavine. Glucose $(20 \mathrm{~mm})$ was added at time point indicated by downward arrow to energize the cells. Efflux of DAPI or Hoechst 33342 is represented by a rapid decrease in the fluorescence, whereas efflux of acriflavine was observed as a rapid increase in the fluorescence. 


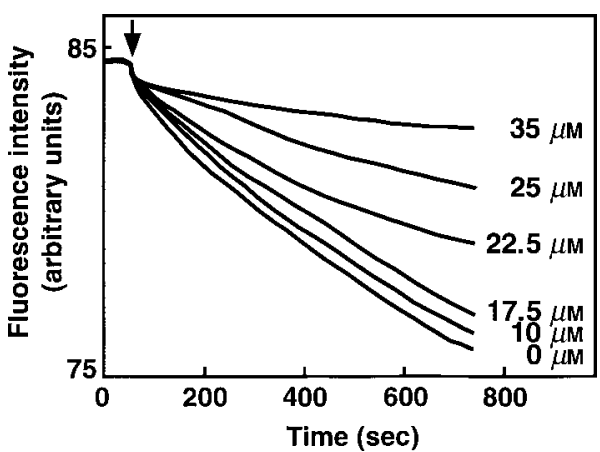

Fig. 3. Inhibition of EmeA-Mediated DAPI Efflux by Reserpine

Energy-starved cells of KAM32/pTEF71 were loaded with DAPI. Cells were resuspended in $0.1 \mathrm{~m}$ MOPS-TMAH (pH 7.0) containing $2 \mathrm{~mm} \mathrm{MgSO}_{4}, 20 \mathrm{~mm} \mathrm{KCl}$ and $5 \mu \mathrm{M}$ DAPI. After incubation for $5 \mathrm{~min}, 20 \mathrm{~mm}$ glucose was added (indicated by an arrow) to energize the cells. Efflux of DAPI from the cells was measured either in the absence or presence of various concentrations $(0$ to $35 \mu \mathrm{M})$ of reserpine.

sponsible for resistance to ethidium bromide, norfloxacin, acriflavine, clindamycin and erythromycin, but not against benzalkonium chloride. On the other hand, EmeA derived from strain ATCC 29212 and expressed in E. coli cells showed resistance to TPPCl, DAPI, Hoechst 33342, norfloxacin, ethidium bromide and benzalkonium chloride, but not against erythromycin. These differences may be due to the distinctiveness of the organisms involved (E. faecalis vs. $E$. coli). It seems possible that E. faecalis cells possess another pump (or other resistance mechanisms) for benzalkonium. Erythromycin is not very effective against $E$. coli because of the presence of an outer membrane, a known permeability barrier for large molecules.

Ethidium bromide is often used as a substrate during the fluorometric assay of multidrug efflux pump activities. In the case of EmeA, it seemed that fluorescent DAPI, Hoechst 33342 and acriflavine were much better substrates (Table 1). Thus, we investigated the efflux properties of these fluorescent antimicrobial agents. We clearly observed an energy-dependent efflux of these compounds. We previously demonstrated that acriflavine was a good substrate for the fluorometric assay of the multidrug efflux pump, VmrA. ${ }^{6}$ We also found that DAPI was a good substrate for the fluorometric assay of multidrug efflux pumps (J. Chen et al., will be published elsewhere). Now we can use these fluorescent probes for the measurement of multidrug efflux pump activities, depending upon their substrate specificities.

Comparison of the deduced amino acid sequences of the two EmeAs revealed that there were differences at 9 positions. These differences may be due to variation in the original strains. The conserved sequences of the 12 putative transmembrane segments (TMS) of the MFS, motifs A, B, C, D2 and $\mathrm{G},{ }^{3)}$ were all conserved well in the EmeA sequence at the corresponding positions (data not shown). Pao et al. ${ }^{35)}$ classified current members of the MFS into 17 (possibly 18) distinct families. Bmr and Blt of B. subtilis and NorA of $S$. aureus, homologues of EmeA from $E$. faecalis, ${ }^{14)}$ were included in family 3, the Drug: $\mathrm{H}^{+}$antiporter (DHA12) family, of the MFS according to this classification. Therefore, it seems that the EmeA protein is a member of the DHA12 group of the MFS judging from the sequence homology and the conserved domains.

Klyachko et al. $^{36)}$ reported that various substitutions of residues Phe-143 and Phe-306 of Bmr not only reduced its sensitivity to reserpine but also significantly changed its substrate specificity, and these two amino acid residues are likely to be involved in substrate recognition. These two Phe residues are conserved at corresponding positions (positions 143 and 315) in the EmeA protein.

Acknowledgements We thank Dr. M. Varela of Eastern New Mexico University for critical reading of the manuscript. This research was supported by a grant from the Ministry of Education, Science, Sport and Culture of Japan.

\section{REFERENCES}

1) Jones R. N., Chest, 119, 397S-404S (2001).

2) Mitscher L. A., Pillai S. P., Gentry E. J., Shankel D. M., Med. Res. Rev., 19, 477-496 (1999).

3) Putman M., van Veen H. W., Konings W. N., Microbiol. Mol. Biol. Rev., 64, 672-693 (2000).

4) Saier M. H., Jr., Tam R., Reizer A., Reizer J., Mol. Microbiol., 11, 841-847 (1994).

5) Levy S. B., Antimicrob. Agents Chemother, 36, 695-703 (1992).

6) Chen J., Morita Y., Huda M. N., Kuroda T., Mizushima T., Tsuchiya T., J. Bacteriol., 184, 572-576 (2002).

7) Huycke M. M., Sahm D. F., Gilmore M. S., Emerg. Infect. Dis., 4, 239-249 (1998).

8) Mundy L. M., Sahm D. F., Gilmore M. S., Clin. Microbiol. Rev., 13, 513-522 (2000).

9) Moellering R. C. J., "Principles and Practices of Infectious Diseases," 4th ed., ed. by Mandell G. L., Bennett J. E., Dolin R., Churchill Livingston, New York, 1995, pp. 1826-1835.

10) Murray B. E., Clin. Microbiol. Rev., 3, 46-65 (1990).

11) Moellering R. C. J., Clin. Infect. Dis., 14, 1173-1178 (1992).

12) Murray B. E., Emerging Infectious Diseases, 4, 37- 47 (1998).

13) Lynch C., Courvalin, P., Nikaido H., Antimicrob. Agents Chemother, 41, 869-871 (1997).

14) Jonas B. M., Murray B. E., Weinstock G. M., Antimicrob. Agents Chemother, 45, 3574-3579 (2001).

15) Lennox E. S., Virology, 1, 190-206 (1955).

16) Berns K. I., Thomas C. A. J., J. Mol. Biol., 11, 117-120 (1965).

17) Sanger F., Nicklen S., Coulson A. R., Proc. Natl. Acad. Sci. U.S.A., 74, 5463-5467 (1977).

18) Japan Society of Chemotherapy., Chemother. (Tokyo), 38, 103-105 (1990).

19) Morita Y., Kataoka A., Shiota S., Mizushima T., Tsuchiya T., J. Bacteriol., 182, 6694-6697 (2000).

20) Kapuscinski J., Szer W., Nucleic Acids Res., 6, 3519—3534 (1979).

21) Putman M., Koole L. A., van Veen H. W., Konings W. N., Biochemistry, 38, 13900-13905 (1999).

22) Tanaka S., Lerner S. A., Lin E. C. C., J. Bacteriol., 93, 642-648 (1967).

23) The Institute for Genomic Research (TIGR) BLAST Search Engine for Unfinished Microbial Genomes: < http://www.tigr.org/>.

24) Eisenberg D., Schwarz E., Komaromy M., Wall R., J. Mol. Biol., 179, 125-142 (1984).

25) Bolotin A., Wincker P., Mauger S., Jaillon O., Malarme K., Weissenbach J., Ehrlich S. D., Sorokin A., Genome Res., 11, 731-753 (2001).

26) Neyfakh A. A., Bidnenko V. E., Chen L. B., Proc. Natl. Acad. Sci. U.S.A., 88, 4781-4785 (1991).

27) Ahmed M., Lyass L., Markham P. N., Taylor S. S., Vazquez-Laslop N., Neyfakh A. A., J. Bacteriol., 177, $3904-3910$ (1995).

28) Yoshida H., Bogaki M., Nakamura S., Ubukata K., Konno M., J. Bacteriol., 172, 6942-6949 (1990).

29) Huda M. N., Morita Y., Kuroda T., Mizushima T., Tsuchiya T., FEMS Microbiol. Lett., 203, 235-239 (2001).

30) Wigler P. W., Patterson F. K., Biochim. Biophys. Acta, 1154, 173-181 (1993).

31) Ahmed M., Borsch C. M., Neyfakh A. A., Schuldiner S., J. Biol. Chem., 268, 11086-11089 (1993).

32) Bolhuis H., van Veen H. W., Brands J. R., Putman M., Poolman B., 
Driessen A. J. M., Konings W. N., J. Biol. Chem., 271, 24123-24128 (1996).

33) Neyfakh A. A., Borsch C. M., Kaatz G. W., Antimicrob. Agents Chemother., 37, 128-129 (1993).

34) Yerushalmi H., Lebendiker M., Schuldiner S., J. Biol. Chem., 270,
6859-6863 (1995).

35) Pao S. S., Paulsen I. T., Saier M. H. J., Microbiol. Mol. Biol. Rev., 62, 1-34 (1998).

36) Klyachko K. A., Schuldiner S., Neyfakh A. A., J. Bacteriol., 179, 2189-2193 (1997). 